\title{
Phyto- und Mykotoxine (1)
}

\author{
Aconitin \\ Jean-Michel Jeannin
}

\section{Aconitum napellus L.; Aconitum species}

A. napellus ist eine mehrjährige krautige Pflanze aus der Familie der Ranunculaceae (Hahnenfussgewächse). Sie erreicht eine Höhe von bis zu $150 \mathrm{~cm}$. Die Wurzelknolle ist verdickt, die Laubblätter sind tief eingeschnitten und gezähnt. Die dunkelblauen oder violetten Blüten stehen in einem traubenartigen Blütenstand. Jeweils das obere der fünf Blütenblätter ist helmförmig ausgebuchtet; der «Helm» ist meistens breiter als hoch $[1,2]$. Die grünen Balgfrüchte tragen die pyramidenförmigen, dreikantigen, geflügelten Samen. Die Pflanze ist in gebirgigen Regionen Mitteleuropas heimisch sowie in Asien, Afrika und Nordamerika eingewandert. In Deutschland, Frankreich, Russland und Spanien wird sie kultiviert [1]. Insgesamt zählt die Gattung über 300 Arten, mit zum Teil ähnlichen, zum Teil unterschiedlichen Wirkungen $[3,4]$. Bekannte Arten sind A. chinense, A. reclinatum, A. lycoctonum mit gelben Blüten und A. uncinatum. Aconitum war während mehrerer Jahre in der USP (U.S. Pharmacopeial Convention) monographiert [1]. A. carmichaelii Debx ist in der Liste der traditionellen asiatischen Substanzen der Swissmedic eingetragen. Die Deutsche Kommission E bewertet Aconitum in ihrer Monographie wegen seiner Toxizität negativ.

\section{Chemie}

A. napellus (Abb. 1) enthält 0,2-2\% Alkaloide, hierunter Aconitin, Picraconitin, Aconine, Napellin [1], Mesaconitin, Lycoctonin, und weitere Terpen-Alkaloide [3]. Der Alkaloidgehalt sinkt mit der Höhe des Standorts: Frische Wurzeln von Pflanzen, die auf einer Höhe von $1750 \mathrm{~m}$ leben, enthalten $0,82 \%$, Wurzeln von Pflanzen, die auf $2500 \mathrm{~m}$ leben, noch $0,29 \%$ [1]. Die oberirdischen Teile enthalten $0,2-2,0 \%$ Alkaloide, die Wurzelknollen bis zu 3\%, die Samen bis zu 1,4\% [3]. Den höchsten Alkaloidgehalt haben Wurzelknollen in der Winterruhe. Weitere Bestandteile sind Aconitinsäure, Itaconsäure, Bern-

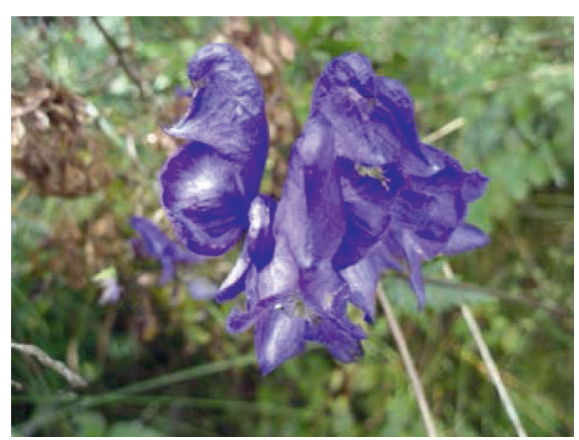

Abb. 1. Aconitum napellus. Unterengadin, 2007.

steinsäure, Äpfelsäure, Fructose, Maltose, Melibiose, Mannitol, Stärke, Fett und Harz [1]. In der Wurzelknolle von A. carmichaelii wurden Aconitin, Coryneinchlorid und Higenamin als herzwirksame Substanzen identifiziert [1]. In älteren Arbeiten werden varietät- bzw. speziesspezifische Aconitinderivate beschrieben: Japaconitin (A. japonicum Fisheri), Indaconitin (A. Chasmanthum, Indischer Eisenhut) und Bikhaconitin (A. spicata Stapf) [4]. Durch Hydrolyse wird Aconitin in Picraconitin umgewandelt; mittels weiterer Hydrolyse wird Aconin gewonnen [1]. Aconitum-Alkaloide ohne Benzoylesterkette (Lappaconitin, N-Deacetyllappaconitin, 6-Benzoylheteratisin, Lappaconidin, Heterasin, Napellin) sind sehr viel weniger toxisch als jene mit einer solchen Kette (Aconitin, Mesaconitin, 3-Acetylaconitin) [5].

\section{Pharmakologie des Aconitins und ausgewählter Derivate}

Aconitin hemmt die neuronale Erregungsübertragung durch Erhöhung der Membranpermeabilität für Natriumionen und lähmt in toxischer Dosierung das Herz und das Atemzentrum. Motorische und sensible Nerven in der Peripherie werden zuerst erregt und später gelähmt [4]. Neuere tierexperimentelle Studien wiesen entzündungshemmende und schmerzstillende Wirkungen für

\section{KARGER}

Fax +497614520714 Information@Karger.com www.karger.com 
Aconitin nach [1]. Auf die Haut aufgetragen ist Aconitin ein Lokalanästhetikum [6]. Extrakte aus A. carmichaelii wirken kardiotonisch. DL-Demethylcoclaurin aus A. carmichaelii steigert die Herzfrequenz bei Sinusarrhythmien. Extrakte bestimmter Spezies haben eine Antitumorwirkung, Extrakte anderer Spezies zeigen antimikrobielle Wirkungen, und wiederum andere Arten wirken antipyretisch [1]. Tinkturen von Aconitum sind lokal zur Behandlung von Trigeminusneuralgien wirksam [7]. Die antinozizeptive Wirkung von Mesaconitin war im Tierexperiment deutlich stärker als diejenige von Morphin. Die Wirkung konnte mit dem Morphinrezeptor-Antagonisten Levallorphan nicht aufgehoben werden. Man vermutet deshalb, dass Mesaconitin an bestimmten Strukturen des Gehirns eingreift. Der antinozizeptive Effekt von 3-Acetylaconitin (aus A. flavum) war im Tierexperiment fünfmal stärker als derjenige von Morphin [5]. Lappaconitin und Mesaconitin zeigten antiepileptische Wirkungen am Hippocampuspräparat. Am Herzen lösen bestimmte Aconitumalkaloide Arrhythmien aus, andere wiederum wirken antiarrhythmisch [5].

\section{Verwendung}

Die innerliche Anwendung von Aconitum ist in der heutigen Medizin nahezu verlassen worden. In den USA werden Extrakte äusserlich für Umschläge verwendet [1]. Aconitum ist im Gegensatz dazu ein weiterhin wichtiges Mittel in der Homöopathie, wo Präparate aus frischem blühendem Aconitum ab der 4. Potenz (D4) zur Behand-

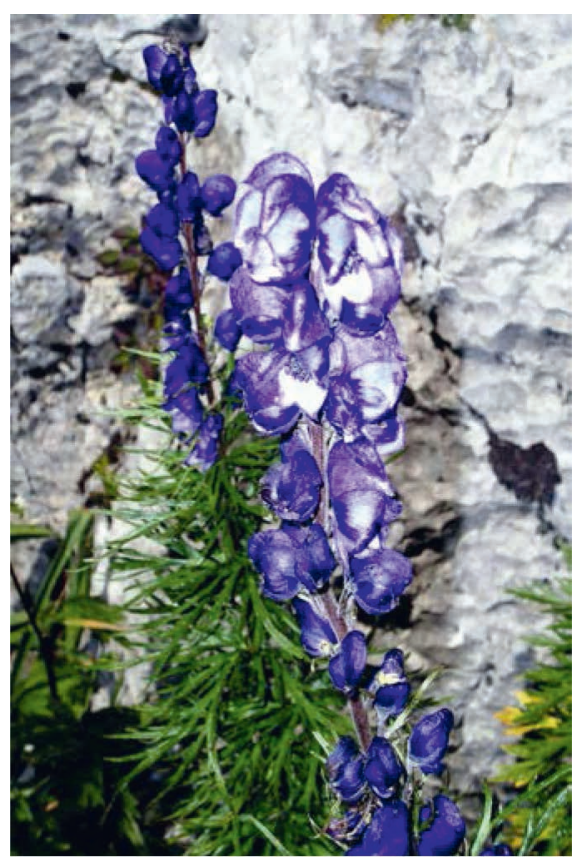

Abb. 2. Aconitum neomontanum Wulfen. Rochers-de-Naye (VD), 2008.

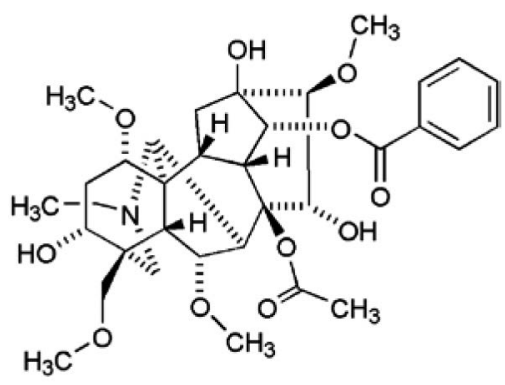

Abb. 3. Aconitin.

Tab. 1. Giftklassen der WHO (nach [3])

\begin{tabular}{lll}
\hline Klasse & Bezeichnung & $\mathrm{LD}_{50}$ Ratte, $\mathrm{mg} / \mathrm{kg} \mathrm{KG}$ \\
\hline Ia & äusserst giftig & $\leq 5$ \\
Ib & sehr giftig & $5-50$ \\
II & giftig & $50-500$ \\
III & schwach giftig & $>500$ \\
\hline
\end{tabular}

lung von Erkältungskrankheiten, Grippe, Neuralgien und Ischias verabreicht werden [8]. In der asiatischen Medizin, namentlich der chinesischen, werden Zubereitungen aus A. chinense Paxt. und A. kusnezoffii Reichb. zur Behandlung der rheumatoiden Arthritis, der chronischen Nierenentzündung, von Ischias und anderer Beschwerden breit eingesetzt. Zubereitungen aus anderen Aconitum-Arten (Abb. 2) sind wegen ihrer analgetischen, antipyretischen, kardiotonischen und antiseptischen Wirkung in der asiatischen Medizin bewährte Mittel. Die Aufbereitung in der asiatischen Medizin unterscheidet sich stark von derjenigen in der europäischen Medizin. Hervorgehoben sei die Behandlung der Droge mit Süssholz, Ingwer und Schwarzen Bohnen. Ausserhalb der Heilkunde wurde A. napellus sehr oft für Giftmorde eingesetzt und auch als Pfeilgift gebraucht [8].

\section{Toxizität}

A. napellus ist als Gift der Klasse Ia klassifiziert (Tab. 1). 10-15 g der getrockneten Wurzelknolle sind für den Erwachsenen tödlich. Die $\mathrm{LD}_{100}$ von Aconitin beträgt für den Menschen 3-6 mg [3].

\section{Intoxikation}

Aconitin (Abb. 3) ist eines der stärksten bekannten natürlichen Gifte. Die Substanz wird über die Schleimhäute und über die intakte Haut gut resorbiert [4]. Symptome einer Vergiftung treten sehr rasch auf: Brennen und Parästhesien im Mund und Rachen, Taubheitsgefühl in Fingern und Zehen, Kribbeln, Ameisenlaufen, Übelkeit, Erbrechen, starke Schmerzen und Krämpfe. Der Tod tritt 0,5-3 h nach der Einnahme durch Atem- oder Herz- 
stillstand ein. Erste-Hilfe-Massnahmen sind Auslösen von Erbrechen, Verabreichung von Natriumsulfat, Kaliumpermanganat und Medizinalkohle [3].

\section{Schlussbetrachtung}

Die Gattung Aconitum ist in mehrfacher Hinsicht exemplarisch: Die Konzentration an Alkaloiden ist von
Organ zu Organ verschieden, hängt von der Höhe des Standorts über dem Meer einer individuellen Pflanze ab und ist saisonal mitbedingt. Aus den verschiedenen Spezies lassen sich Alkaloide isolieren, die gegensätzlich wirken: Die einen lösen Arrhythmien aus, die anderen wirken antiarrhythmisch. Faszinierend ist der Umstand, dass eine Reihe von Substanzen isoliert wurde, die die erwünschten Wirkungen auslösen, jedoch viel weniger toxisch sind als z.B. Aconitin. Ist die Forschung erfolgreich, kann aus der Giftpflanze eine Heilpflanze werden.

\section{Literatur}

1 Leung AY, Forster S: Encyclopedia of Common Natural Ingredients, ed 2. New York, Wiley, 1996.

2 Blauer Eisenhut. http://de.wikipedia.org/ wiki/Blauer_Eisenhut.

3 Wink M, et al: Handbuch der giftigen und psychoaktiven Pflanzen. Stuttgart, WVG, 2008.
Munch JC, et al: Bioassay of aconite and its preparations. 2. The pharmacology and pharmacognosy of various species of aconitum. J Am Pharm Ass 1929;18:986-992.

5 Ameri A: The effects of Aconitum alkaloids on the central nervous system. Progress Neurobiol 1998;56:211-223.
6 Frohe D: Heilpflanzenlexikon, ed 8. Stuttgart, Wissenschaftliche Verlagsgesellschaft, 2006.

7 Aconite; in Hostettmann K: Tout savoir sur les poisons naturels. Lausanne/Paris, Favre, 2006.

8 Eisenhut; in Pahlow M: Das grosse Buch der Heilpflanzen. Augsburg, Bechtermünz, 2000 . 